\title{
X-Ray Stereotactic Lesion Localization in Conjunction With Dedicated Scintimammography
}

Mitali J. Moré, Deepa Narayanan, Patricia J. Goodale, Stan Majewski, Benjamin Welch, Randolph Wojcik, and Mark B. Williams, Member, IEEE

\begin{abstract}
We are developing a dual modality system that combines digital $\mathrm{X}$-ray mammography with gamma emission scintigraphy on an upright mammography gantry. The breast is held under mild compression by a support structure that is independent of the detectors. The $\mathrm{X}$-ray source and detectors can be rotated around a fixed rotation axis permitting multiple views of the breast with fixed compression. Two such views can be combined as a stereotactic pair to obtain the three-dimensional location of breast lesions. Information about the location of the lesion within the breast permits corrections for attenuation and detector spatial resolution, resulting in more accurate estimation of the true lesion-to-background concentration ratio, based on the image lesion-to-background counts ratio. In this paper, we describe the model used to make these corrections, and present the results of the phantom experiments designed to test the accuracy of our calculations.
\end{abstract}

Index Terms-Breast imaging, multimodality, scintimammography, stereotactic localization, technetium-99m sestamibi uptake.

\section{INTRODUCTION}

A CCURATE preoperative staging of breast cancer is required to plan surgery and postoperative therapy. Scopinaro et al. have suggested that the uptake of technetium- $99 \mathrm{~m}$ sestamibi is correlated with angiogenesis and thus can be a potential marker for breast cancer invasiveness [1]. Other researchers have examined the degree of correlation between sestamibi uptake and the traditional indicators of malignancy such as desmoplasia and mitotic activity [2]. All these studies require quantification of the differential radiotracer uptake between the lesion and the surrounding healthy breast tissue. Since the depth of the lesion plays an important role in determining the contrast of the lesion in the image [3], it must be taken into account if accurate uptake values are to be obtained. The attenuation due to the structure overlying the lesion (between the lesion and the camera), and the detector blur, both act to reduce the lesion contrast in the image.

To illustrate the effects of attenuation and detector blur, an image of a phantom containing two simulated lesions is shown

Manuscript received January 28, 2003; revised June 9, 2003. This work was supported in part by the National Institutes of Health Grants RO1 CA69452 and RO1 CA66232, the U.S. Army Breast Cancer Research Program Grant BC980469, and the Susan G. Komen Foundation Grant 99-003050. The Southeastern Universities Research Association (SURA) operates the Jefferson Lab for the U.S. Department of Energy under contract DE-AC05-84ER40150.

M. J. Moré, D. Narayanan, P. J. Goodale, and M. B. Williams are with the University of Virginia, Charlottesville, VA 22908 USA (e-mail: mjm4j@virginia.edu).

S. Majewski, B. Welch, and R. Wojcik are with the Thomas Jefferson National Accelerator Facility, Newport News, VA 23606 USA.

Digital Object Identifier 10.1109/TNS.2003.817303

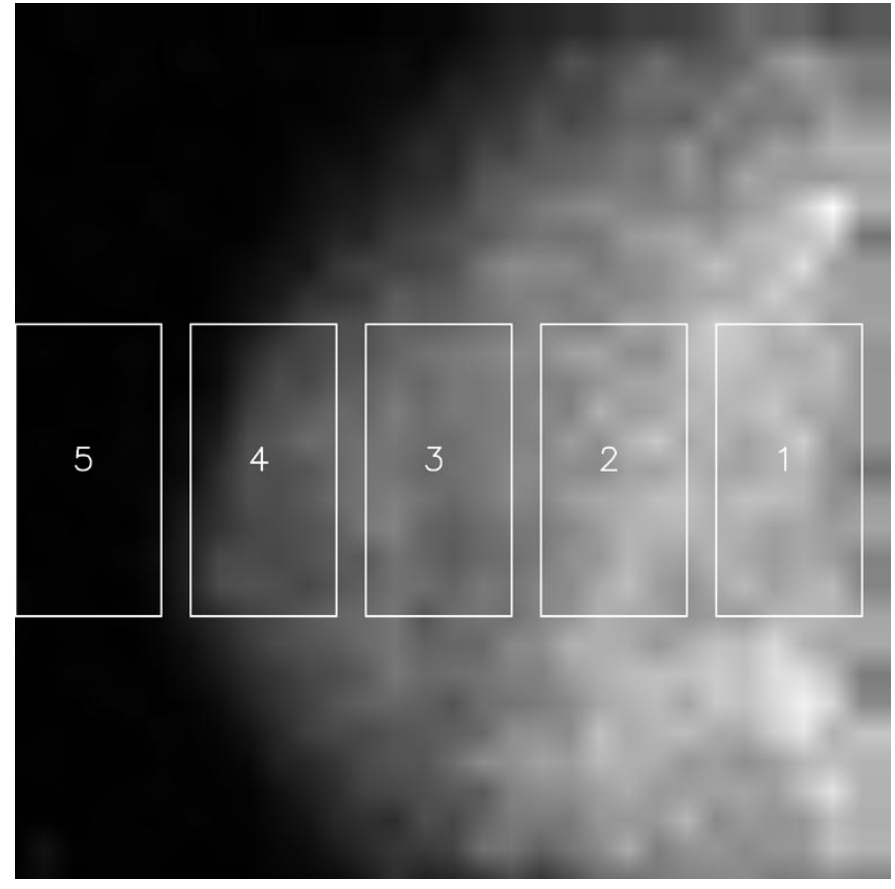

Fig. 1. Image showing 2 lesions of equal size and equal radioactivity concentration but placed at varying depth from the detector surface. The image contrast for lesion 1 is 0.26 and lesion 2 is 0.42 . The true concentration ratio between the lesions and the background was $10: 1$.

in Fig. 1. The image shows two lesions of equal size (inner diameter $=1 \mathrm{~cm})$ and equal radioactivity concentration $(3 \mu \mathrm{Ci} / \mathrm{cc})$ but at different depths in the phantom, and therefore different distances from the detector. The background radioactivity concentration was $0.3 \mu \mathrm{Ci} / \mathrm{cc}$. Lesion 1 is at a distance of $7.7 \mathrm{~cm}$ from the detector whereas lesion 2 is at a distance of $5 \mathrm{~cm}$. Even though the lesions have equal size and radioactivity concentration, their image contrast is vastly different. The image contrast for lesion 1 is 0.26 whereas that for lesion 2 is 0.42 . This illustrates the fact that in order to quantify the radiotracer concentration, it is necessary to make corrections to the lesion-to-background contrast observed in the image.

We are developing a dual modality breast scanner with a digital X-ray detector and a gamma-ray detector mounted on a single upright mammography unit. In the first generation of the scanner, the surface of the X-ray detector acts as the breast support [4]. Mild compression is applied and an X-ray and a gamma image are obtained. Merging the two images together provides structural and functional information in a single image. The second generation dual modality breast scanner is designed to permit multiple views of the breast with fixed compression. 


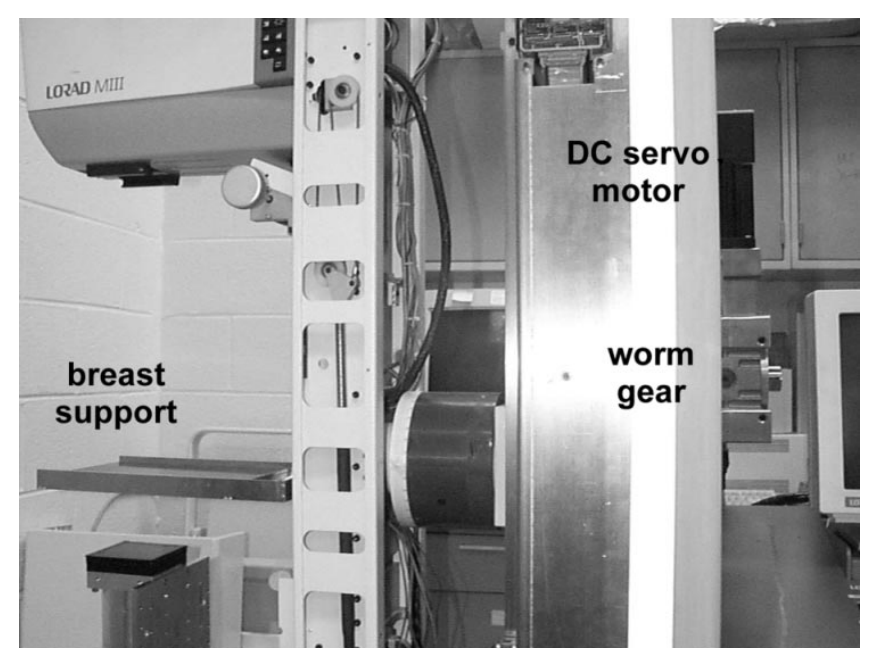

Fig. 2. Photograph of the dual modality system equipped with breast support structure independent of the X-ray detector. A small field of view X-ray detector is shown mounted on the gantry. However, the gamma camera is not included in the picture.

The breast is held under mild compression on a breast support structure that is independent of the detectors and the gantry arm [5]. The X-ray source and the X-ray and gamma detectors are mounted on a motor-driven gantry arm. The motorized gantry with the breast support structure and a small field of view X-ray detector mounted on it is shown in Fig. 2. The mechanical aspects of this system have been described earlier [5]. Multiple view imaging makes it possible to obtain three-dimensional lesion localization with this system.

\section{STEREOTACTIC LocAliZATION}

Lesion localization via stereotactic imaging is a method commonly used in breast needle biopsies [6]-[8]. The location of the lesion in the two stereo images is used to calculate the three-dimensional location of the lesion center, $\mathrm{x}_{\mathrm{O}}, \mathrm{y}_{\mathrm{o}}, \mathrm{z}_{\mathrm{O}}$. The expressions for $x_{0}, y_{0}, z_{0}$ are given in (1)-(3), shown at the bottom of the page. The derivation of these equations and definition of the coordinate system and variables used are included in the Appendix.

\section{THEORY}

\section{A. Background Concentration}

In the gamma emission image of the breast obtained in a dual modality scan, the average number of primary counts (i.e., not including scatter) per pixel $\left(\mathrm{N}_{\mathrm{b}}\right)$ in the background region of the image (i.e., a region of the breast adjacent to the lesion) can be calculated by integrating the activity along the thickness of the breast

$$
N_{b}=\varepsilon_{t p}^{2} \int_{0}^{D} C_{b}(z) e^{-\mu_{t} z} d z
$$

where $\varepsilon$ is the detection efficiency, $t$ is the acquisition time, $p$ is the pixel size, and $\mathrm{C}_{\mathrm{b}}(\mathrm{z})$ is the activity per unit volume in the background portion of the breast. $\mathrm{D}$ is the thickness of the breast above the pixel and $\mu_{\mathrm{t}}$ is the linear attenuation coefficient of the breast tissue for $140 \mathrm{keV}$ gamma-rays. Since mild breast compression is used, D is simply the compressed breast thickness. In the case where the background activity concentration is uniform along the line of integration, this equation can easily be rearranged to calculate the background concentration

$$
C_{b}=\frac{N_{b} \mu_{t}}{\varepsilon t p^{2}\left[1-e^{-\mu_{t} D}\right]} .
$$

The average number of counts obtained in a background pixel of the image is the sum of the scatter and the primary counts, $\mathrm{N}_{\mathrm{b}}$. These scatter counts must be subtracted from the sum to obtain the primary background counts. The scatter-to-primary ratio (SPR) is dependent on the location within the breast, increasing with decreasing distance from the chest wall [9]. In practice, the position-dependent SPR is obtained by acquiring images in list mode. This permits retrospective computation of position-dependent pulse height spectra from which the SPR can be obtained.

\section{B. Lesion Concentration}

There are two primary factors that make the contrast of the lesion in the image a poor measure of the relative radiotracer concentrations in the lesion and the background breast tissue. These are: i) the attenuation of gamma-rays originating in the lesion, caused by the tissue overlying the lesion and ii) the blur due to the detector point spread function (PSF). In addition, for both lesion pixels and background pixels, scatter adds additional counts thus reducing the apparent concentration ratio in the image.

The lesion concentration is given by

$$
C_{L}=\frac{N_{\mathrm{L}, \text { total }}}{V_{L} t \varepsilon A}
$$

where $\mathrm{N}_{\mathrm{L} \text {, total }}$ is the total number of detected counts from the lesion, $\mathrm{V}_{\mathrm{L}}$ is the lesion volume, $\mathrm{t}$ is the acquisition time, $\varepsilon$ is the gamma camera efficiency and $\mathrm{A}$ is the attenuation of the breast tissue overlying the lesion. The amount of attenuation is given by:

$$
A=e^{-\mu_{t} b}
$$

$$
\begin{aligned}
x_{o} & =\frac{(a-c) \sin \theta\left(\left(z_{1} x_{2}+z_{2} x_{1}\right)+(a-c)\left(\sin \theta\left(z_{1}-z_{2}\right)-\cos \theta\left(x_{1}+x_{2}\right)\right)\right)}{\left(z_{1} x_{2}-z_{2} x_{1}\right)+(a-c)\left(\sin \theta\left(z_{1}+z_{2}\right)+\cos \theta\left(x_{1}-x_{2}\right)\right)-2(a-c)^{2} \sin \theta \cos \theta} \\
z_{o} & =\frac{x_{o} z_{1}-(a-c) x_{o} \cos \theta-(a-c) z_{1} \sin \theta+(a-c) x_{1} \cos \theta}{x_{1}-(a-c) \sin \theta} \\
y_{o} & =\frac{L_{y 1}\left(x_{o}-(a-c) \sin \theta\right)}{L_{x 1} \cos \theta+x_{d 1}-(a-c) \sin \theta} .
\end{aligned}
$$


where $b$ is the depth of the lesion in the breast.

The blurring due to the detector PSF does not change the total number of counts detected from the lesion but only redistributes these counts over a larger area thereby reducing the amplitude of the lesion peak in the image. The projection of the spherical lesion on a perfect detector (that is a detector whose PSF is a delta function) can be expressed as

$$
\begin{aligned}
N_{L}(x, y)= & N_{L, \max }\left\{1-\left[\frac{\left|x-x_{o}\right|^{2}+\left|y-y_{o}\right|^{2}}{R^{2}}\right]\right\}^{1 / 2} \\
& \text { for } \frac{\left|x-x_{o}\right|^{2}+\left|y-y_{o}\right|^{2}}{R^{2}}<1 \\
= & 0 \text { for } \frac{\left|x-x_{o}\right|^{2}+\left|y-y_{o}\right|^{2}}{R^{2}} \geq 1
\end{aligned}
$$

where $\mathrm{R}$ is the lesion radius, $\mathrm{x}_{\mathrm{O}}$ and $\mathrm{y}_{\mathrm{O}}$ are the $\mathrm{x}$ and $\mathrm{y}$ coordinates of the lesion center and the peak amplitude $\mathrm{N}_{\mathrm{L}}$, max is set by the condition

$$
\iint N_{L}(x, y) d x d y=N_{\mathrm{L}, \text { total }}
$$

To calculate the reduction in the amplitude at the lesion center in the image due to the PSF of the detector, the two-dimensional projection of the spherical lesion on the detector given by (8) is convoluted with the two-dimensional Gaussian PSF of the detector. The width of the Gaussian in both dimensions is a function of the distance of the source from the detector surface. The detector PSF, normalized for peak amplitude of unity, can be written as

$$
\begin{aligned}
g(x, y) & =\exp \left(-\frac{\left(x-x_{o}\right)^{2}}{2 \sigma_{x}^{2}}-\frac{\left(y-y_{o}\right)^{2}}{2 \sigma_{y}^{2}}\right) \\
N_{\mathrm{L}, \text { blur }}(x, y) & =N_{\mathrm{L}}(x, y) \otimes g(x, y) .
\end{aligned}
$$

Convoluting the above two functions, we get the blurred spatial distribution of the detected lesion counts in the image, $\mathrm{N}_{\mathrm{L} \text {, blur }}(\mathrm{x}, \mathrm{y})$. Clearly, $\mathrm{N}_{\mathrm{L} \text {, blur }}(\mathrm{x}, \mathrm{y})$ is spread over a larger area and has a reduced height relative to the original lesion projection, $\mathrm{N}_{\mathrm{L}}(\mathrm{x}, \mathrm{y})$. The ratio of the peak amplitude of $\mathrm{N}_{\mathrm{L} \text {, blur }}(\mathrm{x}, \mathrm{y})$ to that of $\mathrm{N}_{\mathrm{L}}(\mathrm{x}, \mathrm{y})$ is defined as the contrast reduction coefficient (CRC) [3]

$$
C R C=\frac{N_{\mathrm{L}, \text { blur, } \max }}{N_{\mathrm{L}, \max }} .
$$

The detector blur correction is applied by dividing the amplitude of the lesion profile in the image, $\mathrm{N}_{\mathrm{L}}$, blur, max by the CRC

$$
N_{\mathrm{L}, \max }=\frac{N_{\mathrm{L}, \text { blur, } \max }}{\mathrm{CRC}} \text {. }
$$

We thus obtain the amplitude of the lesion profile in the absence of detector blur, $\mathrm{N}_{\mathrm{L} \text {, max }}$ and can calculate the total number of detected lesion counts, $\mathrm{N}_{\mathrm{L} \text {, total }}$ from (8) and (9) and finally the lesion concentration, $C_{L}$ from (6).

\section{Phantom Measurements}

\section{A. Lesion Localization With $X$-ray}

A fillable phantom of rectangular cross section was designed to simulate a compressed breast. A tube was located at the phantom center to which spherical lesions were attached using low attenuation rods of known length, $r$, oriented at a known

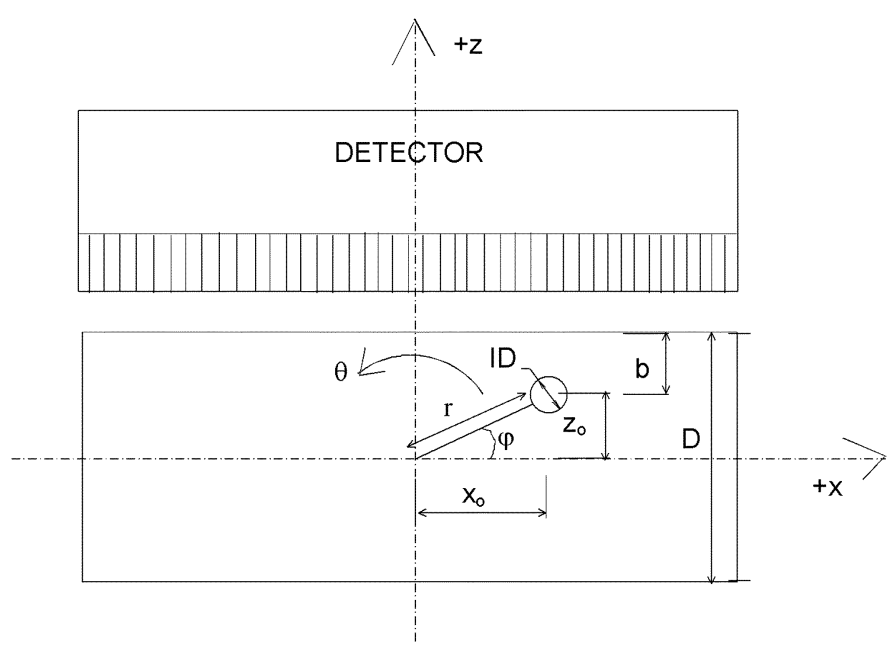

Fig. 3. Experimental setup used for the phantom measurements. A rectangular box phantom was used. The central tube with lesions attached by low attenuation rods was rotated independent of the outer box. Based on the known values of $r$ and $\varphi$, the three-dimensional location of each lesion for each $\theta$ value could be calculated.

angle, $\varphi$ with respect to the phantom surface. A support was designed to allow the phantom to be mounted with the tube on the rotation axis of the gantry. A bearing allowed the gantry arm to rotate while the phantom was held stationary. This setup enabled us to place the lesions at accurately known $\mathrm{x}, \mathrm{y}$ and $\mathrm{z}$ coordinates. Lesions of interior diameter 0.8 and $1.0 \mathrm{~cm}$ from Data Spectrum Corp., Chapel Hill, NC, were used for this study. The wall thickness of the lesions was only $\sim 1 \mathrm{~mm}$ and hence the attenuation through the walls could be ignored for the concentration calculation. With the phantom positioned independently of the X-ray detector, images were acquired at several different angles from $-90^{\circ}$ to $+90^{\circ}$. The phantom was filled with water and the lesions contained a small amount of gadolinium-DTPA for enhanced X-ray contrast.

\section{B. Concentration Ratio Measurement}

For simplicity, acquisition of the gamma images of the phantom was performed with the gamma camera positioned stationary on a bench top. A schematic of the experimental setup is shown in Fig. 3. The lesions and background were filled with a solution of technetium-99m with a lesion-to-background concentration ratio of $10: 1$ to simulate hot lesions in a warm background. The absolute concentration in the background was $0.48 \mu \mathrm{Ci} / \mathrm{cc}$ and that in the lesion was $4.9 \mu \mathrm{Ci} / \mathrm{cc}$. In order to test our ability to measure concentration ratios over a range of lesion depths, the central tube of the phantom was attached to a rotation stage from Velmex, Inc., Bloomfield, NY (website: http://www.velmex.com), and rotated independently of the fixed phantom housing over a range of $360^{\circ}$. At each angle, $\theta$, the coordinates $\mathrm{x}_{\mathrm{O}}, \mathrm{y}_{\mathrm{o}}, \mathrm{z}_{\mathrm{O}}$ of the lesion centers were known based on the known rod lengths and angular spacing between lesions. Images were acquired every $15^{\circ}$ over a range of $360^{\circ}$. Rotating the tube with respect to the phantom housing and detector surface places the lesions at varying depth and lesion-to-collimator distance, thereby permitting the correction model to be tested over a range of lesion depths with a single setup. 


\section{Detector Characterization}

The gamma camera used for the studies described here consists of a $4 \times 4$ array of R7600-00-C8 position sensitive photomultiplier tubes (PSPMT's) from Hamamatsu, Bridgewater, NJ. The PSPMT's are optically coupled via acrylic light guides to a $30 \times 30$ array of $\mathrm{NaI}(\mathrm{Tl})$ crystals from Saint-Gobain, Newbury, Ohio. Each crystal is $3 \mathrm{~mm} \times 3 \mathrm{~mm} \times 6 \mathrm{~mm}$ (thick), and the crystal center-to-center spacing is $3.3 \mathrm{~mm}$. The active field of view of the gamma camera is $\sim 10 \mathrm{~cm} \times 10 \mathrm{~cm}$ and the energy resolution is $15 \%$ at $140 \mathrm{keV}$. A lead collimator with $1.58 \mathrm{~mm}$ hexagonal apertures and height of $21 \mathrm{~mm}$ was used. The septal thickness was $0.267 \mathrm{~mm}$. An energy window of $-7 \% /+22 \%$ centered around the $140 \mathrm{keV}$ photopeak was used. The camera sensitivity with this energy window is $220 \mathrm{cpm} / \mu \mathrm{Ci}$ (efficiency of $\left.1.0 \times 10^{-4}\right)$. Further details on the detector design and performance are published elsewhere [10].

The full width at half maximum (FWHM) of the two-dimensional gamma camera PSF was obtained by measuring the image of a ${ }^{57} \mathrm{Co}$ spot marker from Isotope Products, Valencia, $\mathrm{CA}$, at a variety of source-to-collimator distances. For each source-to-collimator distance, the spot marker, which has an active diameter of $\sim 1.5 \mathrm{~mm}$, was imaged at several locations on the detector surface. These images were shifted and summed to obtain a source image profile averaged over the camera surface. The profile of the point source, which was assumed to be a uniform disc of diameter $1.5 \mathrm{~mm}$, was deconvoluted from the averaged profile. The result was then fitted with a two-dimensional Gaussian function. The FWHM of the fitted function in the $\mathrm{x}$ - and $\mathrm{y}$-dimensions are plotted as a function of the source to detector (source to crystal) distance in Fig. 4.

\section{DATA ANALYSIS}

\section{A. Lesion Localization}

An analysis program written in IDL (Interactive Data Language from Research Systems, Inc., Boulder, CO, http://www.rsinc.com) was used to locate the lesion center in the stereo images. Using (1)-(3) the coordinates of the lesion center were calculated. The $\mathrm{z}$ value of the lesion was then used to calculate the lesion and background concentration from the image counts.

\section{B. Concentration Ratio Measurement}

1) Background Concentration: To get the number of background counts, a profile through the gamma image is taken which does not contain any lesions. For each lesion, profiles are extracted from rows near that lesion. From these rows, the average number of background counts is obtained using pixels taken from columns containing the lesion.

The scatter contribution to this background pixel count is subtracted to obtain the primary counts in the background. For the phantom used here, the scatter fraction is approximately constant and uniform throughout the region in which the lesions are located. The technique for obtaining the scatter fraction for a box phantom such as that used in these experiments has been described earlier [5]. The scatter fraction for the energy window $-7 \% /+22 \%$ was measured to be 0.2 . The background concen-

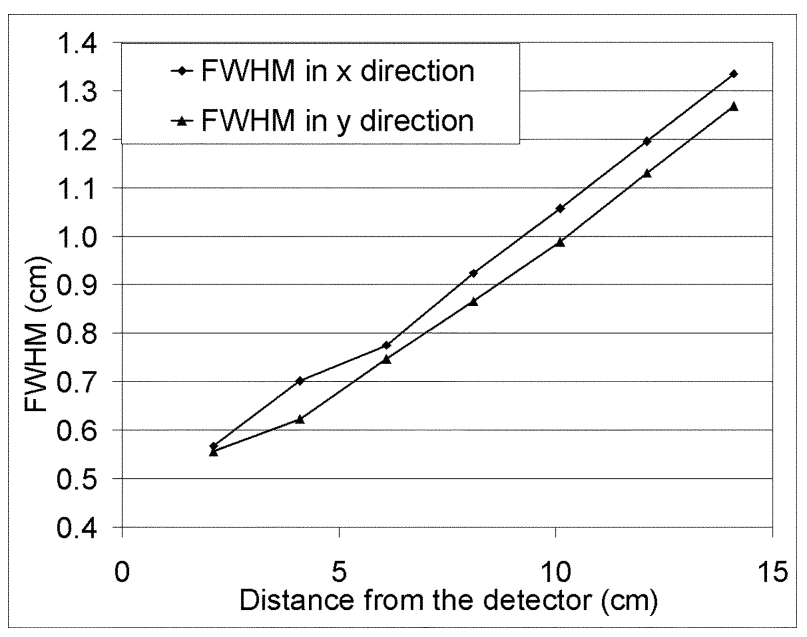

Fig. 4. Plot of the FWHM of the detector measured in two dimensions versus the distance from the detector.

tration is then determined from the primary background counts as described in (5).

2) Lesion Concentration: To calculate the lesion concentration, a region of interest centered on the lesion is first extracted. The number of counts in a pixel imaging the lesion is the sum of lesion counts and counts from the background volume above and below the lesion. The average number of background counts must be subtracted from the total number of lesion pixel counts to obtain the lesion-only counts. In order to obtain a good estimate of the amplitude of the lesion profile in the presence of statistical fluctuations, a two-dimensional surface is first fitted to the background subtracted lesion profile. This lesion profile amplitude must be divided by the CRC to compensate for the detector blur.

To obtain the CRC the following procedure is used. The known lesion-to-collimator distance is used to determine the $\mathrm{x}$ and y-widths of the Gaussian detector PSF (Fig. 4). The PSF is convoluted with the theoretical lesion projection as in (11) and the theoretical CRC is obtained. The known lesion radius was used for the value of $\mathrm{R}$ in (8). The peak height obtained after dividing the fitted lesion amplitude by the CRC is then attributed to the maximum of the unblurred lesion projection function, $\mathrm{N}_{\mathrm{L}}(\mathrm{x}, \mathrm{y})$, and the area under it is calculated to obtain the total counts coming from the lesion.

These counts are then divided by the attenuation of the overlying tissue. For the case of the phantom study reported here, the overlying tissue is water and the phantom wall. The attenuation of the gamma-rays from the lesion is given by:

$$
A=e^{-\mu_{w} b} A_{\text {wall }}
$$

where $\mu_{\mathrm{w}}$ is the linear attenuation coefficient of water for 140 $\mathrm{keV}$ gamma-rays and $A_{\text {wall }}$ is the attenuation of the acrylic wall, on the camera side of the phantom.

\section{RESULTS}

\section{A. Lesion Localization}

Table I shows the results obtained for the stereotactic localization using the $\mathrm{X}$-ray imaging component of the dual modality system. These were obtained with stereo views at angles $+15^{\circ}$ 
TABLE I

MEASURED AND CALCUlated Lesion LOCATIONS

\begin{tabular}{c|cc|cc|cc}
\hline Lesion \# & Known & & Calculated & Difference & \\
& xo $(\mathrm{cm})$ & zo $(\mathrm{cm})$ & xo $(\mathrm{cm})$ & zo $(\mathrm{cm})$ & x $(\mathrm{mm})$ & $\mathrm{z}(\mathrm{mm})$ \\
\hline 1 & -1.00 & 2.00 & -0.99 & 2.04 & -0.08 & -0.39 \\
2 & 4.00 & 1.00 & 4.02 & 0.96 & -0.23 & 0.44 \\
3 & 2.00 & -1.00 & 2.00 & -1.04 & 0.00 & 0.41 \\
4 & -3.00 & 0.00 & -3.01 & 0.05 & 0.14 & -0.48 \\
\hline
\end{tabular}

and $-15^{\circ}$. Columns 2 and 3 give the known location of the lesion center in the $\mathrm{x}$ and $\mathrm{z}$ dimension and columns 4 and 5 give their location. The last column gives the error in millimeters, obtained by subtracting the calculated values from the known values. Similar results were obtained using other stereo angles up to $\pm 60^{\circ}$.

\section{B. Concentration Ratio Measurement}

Fig. 5 shows the ratios between the concentrations in two of the simulated lesions and the background, plotted as a function of the rotation angle of the central tube. Both lesions had an inner diameter of $1.0 \mathrm{~cm}$ and a lesion to background ratio of $10: 1$. Plots are shown of concentration ratios calculated both with and without corrections for detector blur, attenuation, and scatter. Corrected ratios were calculated using the method described above. Uncorrected concentration ratios were obtained for each view (all $\theta$ values) as follows. ROIs were drawn containing the lesion image, and in a region nearby containing only background. The average background count per pixel was subtracted from each pixel in the lesion ROI. A smooth two-dimensional function was fitted to the resulting lesion profile, and its area was calculated. This area, divided by the known lesion volume, was taken as the lesion concentration. The background concentration was taken to be the total number of counts in the background ROI divided by the product of the ROI area and the phantom thickness. Fig. 5 shows that with corrections made, the concentration ratios are closer to the true concentration ratio.

Plot 6 shows, for every $\theta$, the image contrast obtained using the measured lesion and background pixel counts, without any corrections made for attenuation or resolution degradation (squares). This illustrates that estimation of concentration ratios based simply on image contrast can be subject to large errors.

\section{DISCUSSION AND CONCLUSION}

The results obtained for the stereotactic lesion localization demonstrate accuracy to within $1-2 \mathrm{~mm}$. This is accurate enough to be able to use the information to make corrections for the attenuation and detector blur and to significantly improve our estimate of the true concentration ratio in the tumor and the background, as is demonstrated in Fig. 6. However, for this phantom study, our calculations used the known lesion radius and volume. In the clinical situation, these parameters are unknown, and must be estimated from the images. Hence, methods need to be developed to extract this information from the high-resolution X-ray image. Similarly, the scatter fraction in the breast is a parameter that must be measured. The scatter fraction is a function of the location in the breast, and increases with decreasing distance to the chest wall [9]. Position-dependent energy spectra are routinely obtained during a dual

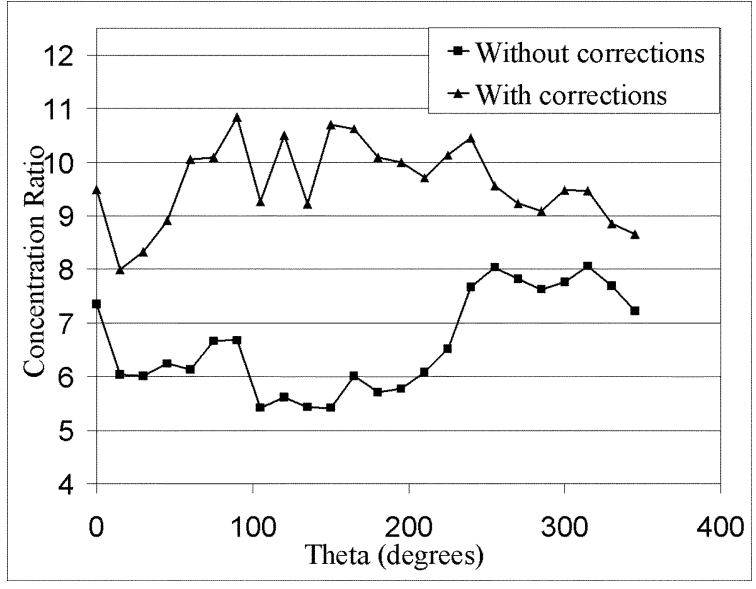

(a)

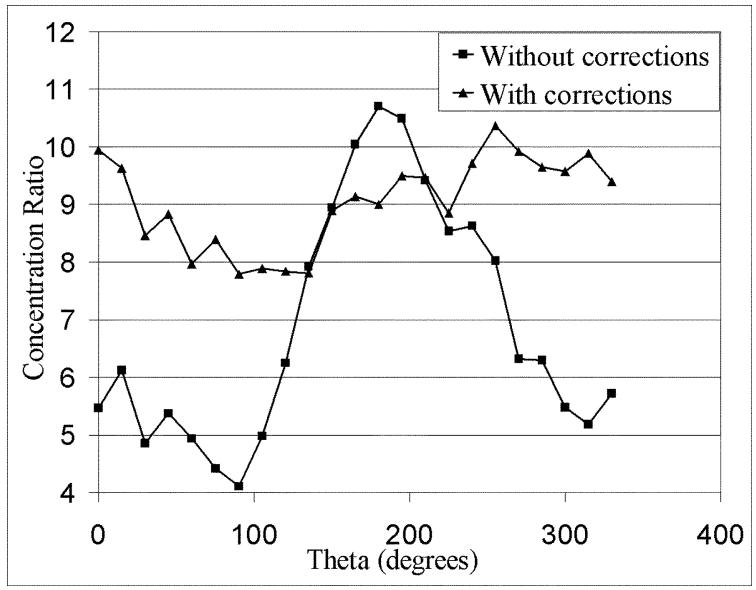

(b)

Fig. 5. Concentration ratios calculated with and without corrections plotted as a function of the rotation angle for two lesions with different $\mathrm{r}(\mathrm{r}=1.4 \mathrm{~cm}$ in the upper graph and $\mathrm{r}=2.7 \mathrm{~cm}$ in the lower graph). The difference in the depth variation $(2.8 \mathrm{~cm}$ versus $5.4 \mathrm{~cm})$ is reflected in the difference in the shapes of the uncorrected curves.

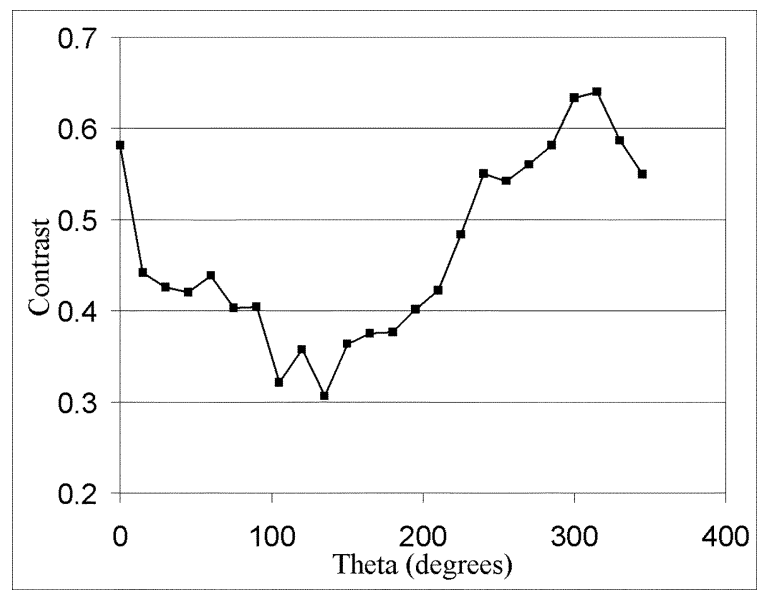

Fig. 6. Image contrast versus rotation angle for the lesion whose concentration ratio is shown in the upper graph of Fig. 5.

modality scan, and can be used to obtain the position dependent scatter fraction.

In this paper we have described lesion localization using $\mathrm{X}$-ray imaging. In principle, the three-dimensional lesion location could also be obtained using gamma-ray stereo 


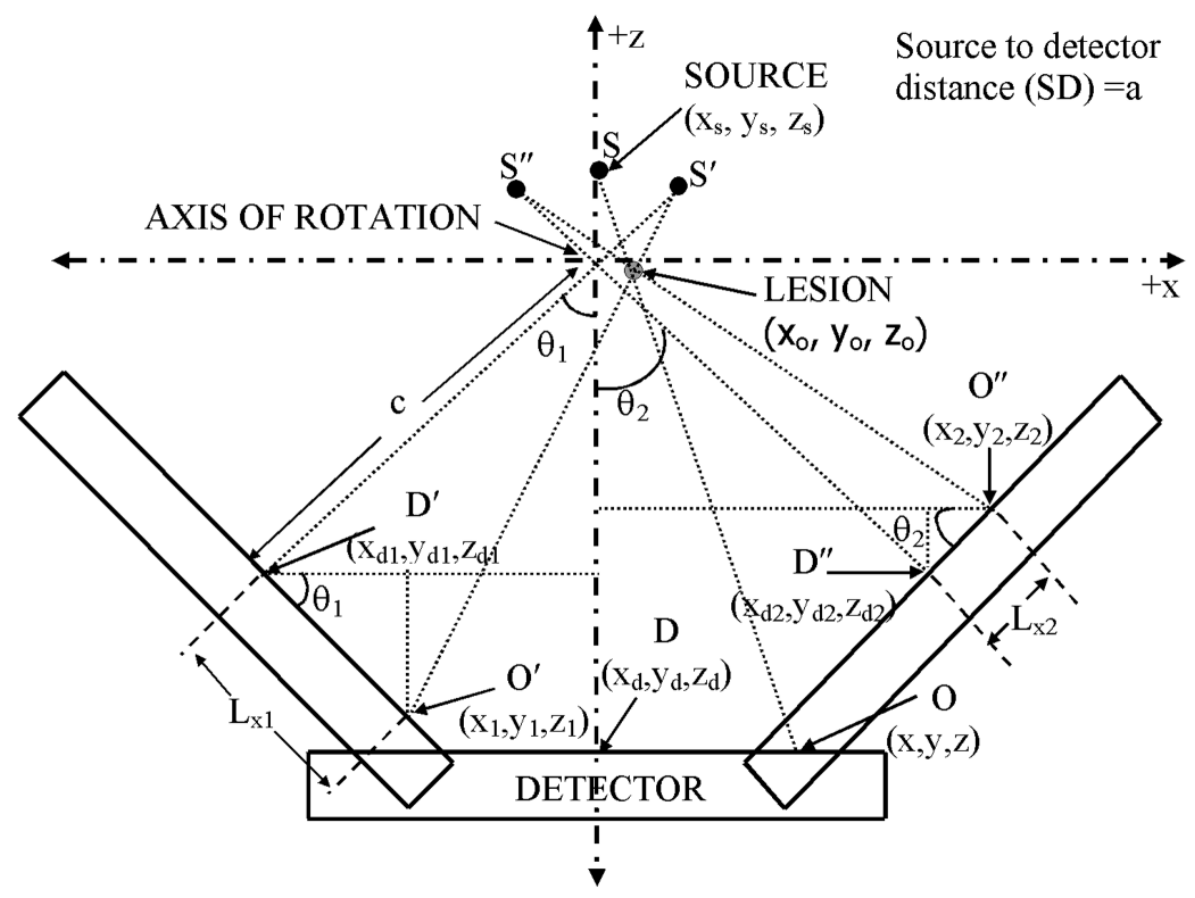

Fig. 7. Schematic of the coordinate system and setup used to derive the equations for lesion localization as viewed along the $+y$ axis.

imaging [11]. However, gamma image acquisition times are significantly longer than those of X-ray images, and the accuracy of localization is lower due to the lower resolution of the gamma detectors. Also, it is desirable to have two X-ray views in order to better estimate the lesion shape and volume. We have therefore elected to use stereotactic X-ray imaging for lesion localization rather than gamma imaging. However, in situations where the lesion is poorly visualized radiographically (e.g., radiodense breasts), but is well visualized in the gamma images, stereotactic gamma localization would be the approach of choice.

Our phantom studies suggest that with appropriate corrections for attenuation, detector blur and scatter, based on measured lesion location, we can more accurately predict radioactivity concentration ratios. Stereotactic X-ray imaging combined with gamma-ray imaging is a potentially powerful method to quantify radiotracer uptake in breast tumors.

\section{APPENDIX}

Following is the derivation of (1)-(3), which calculate the coordinates of the lesion center based on two stereo views. The coordinate system is shown in Fig. 7. We define the origin of the coordinate system to be the point on the rotation axis that is intersected by the line between the X-ray focal spot and the center point of the chest wall edge of the X-ray detector. The $\mathrm{x}$-axis is defined to lie along the medial-to-lateral direction of the patient, with positive $\mathrm{x}$ pointing toward the right of the patient. The $y$-axis is along the rotation axis of the gantry with the positive direction going toward the gantry and away from the patient. The z-axis is defined to be along the detector to source direction when the gantry arm is positioned vertically, with positive $\mathrm{z}$ going toward the source. Let $a$ be the source to detector distance and $c$ be the distance between the origin and the center point of the chest wall edge of the detector surface.

As shown in Fig. 7, $\left(\mathrm{x}_{\mathrm{s}}, \mathrm{y}_{\mathrm{s}}, \mathrm{z}_{\mathrm{s}}\right)$ are the coordinates of the source, $\mathrm{S}$, when the gantry arm is vertical $(\theta=0)$ and $\left(\mathrm{x}_{\mathrm{d}}, \mathrm{y}_{\mathrm{d}}\right.$, $z_{d}$ ) are the coordinates of the detector chest wall edge center, $\mathrm{D}$, when $\theta=0 . \mathrm{O}(\mathrm{x}, \mathrm{y}, \mathrm{z})$ is the projection of the center of the lesion with spatial coordinates $\left(\mathrm{x}_{\mathrm{O}}, \mathrm{y}_{\mathrm{o}}, \mathrm{z}_{\mathrm{o}}\right)$ on the detector plane.

If the source and detector are rotated through an angle $\theta_{1}$, the new source and detector center coordinates are $\mathrm{S}^{\prime}\left(\mathrm{x}_{\mathrm{s} 1}, \mathrm{y}_{\mathrm{s} 1}, \mathrm{z}_{\mathrm{s} 1}\right)$ and $\mathrm{D}^{\prime}\left(\mathrm{x}_{\mathrm{d} 1}, \mathrm{y}_{\mathrm{d} 1}, \mathrm{z}_{\mathrm{d} 1}\right)$ respectively, and the image of the lesion center on the detector is located at $\mathrm{O}^{\prime}\left(\mathrm{x}_{1}, \mathrm{y}_{1}, \mathrm{z}_{1}\right)$. We denote the perpendicular distance in the images between the lesion center and the projection of the rotation axis onto the detector by $\mathrm{L}_{\mathrm{x} 1}$, and denote the perpendicular distance between the lesion center and the chest wall edge of the detector by $\mathrm{L}_{\mathrm{y} 1}$. From geometry we get the following relationships:

$$
\begin{aligned}
x_{s 1} & =(a-c) \sin \theta_{1} ; \quad y_{s 1}=0 ; \quad z_{s 1}=(a-c) \cos \theta_{1} \\
x_{d 1} & =-c \sin \theta_{1} ; \quad y_{d 1}=0 ; \quad z_{d 1}=-c \cos \theta_{1} \\
x_{1} & =L_{x 1} \cos \theta_{1}+x_{d 1} ; \quad y_{1}=L_{y 1} ; \quad z_{1}=z_{d 1}-L_{x 1} \sin \theta_{1} .
\end{aligned}
$$

The equation of line $\mathrm{S}^{\prime} \mathrm{O}^{\prime}$ is given by

$$
\begin{aligned}
& \frac{x_{o}-x_{s 1}}{x_{1}-x_{s 1}}= \frac{y_{o}-y_{s 1}}{y_{1}-y_{s 1}}=\frac{z_{o}-z_{s 1}}{z_{1}-z_{s 1}} \\
& \frac{x_{o}-(a-c) \sin \theta_{1}}{x_{1}-(a-c) \sin \theta_{1}}=\frac{z_{o}-(a-c) \cos \theta_{1}}{z_{1}-(a-c) \cos \theta_{1}} \\
& \text { Let } R=(a-c) \sin \theta_{1} \\
& \quad \text { and } T=(a-c) \cos \theta_{1}
\end{aligned}
$$

then

$$
\begin{aligned}
\frac{x_{o}-R}{x_{1}-R} & =\frac{z_{o}-T}{z_{1}-T} \\
z_{o} & =\frac{\left(x_{o}-R\right)\left(z_{1}-T\right)}{x_{1}-R}+T .
\end{aligned}
$$




$$
\begin{aligned}
& x_{o}=\frac{R\left(z_{1}-T\right)\left(x_{2}-S\right)-S\left(z_{2}-U\right)\left(x_{1}-R\right)+(T-U)\left(x_{1}-R\right)\left(x_{2}-S\right)}{-\left(z_{2}-U\right)\left(x_{1}-R\right)+\left(z_{1}-T\right)\left(x_{2}-S\right)} \\
& z_{o}=\frac{\left(x_{o}-R\right)\left(z_{1}-T\right)}{\left(x_{1}-R\right)}+T . \\
& x_{o}=\frac{(a-c) \sin \theta\left(\left(z_{1} x_{2}+z_{2} x_{1}\right)+(a-c)\left(\sin \theta\left(z_{1}-z_{2}\right)-\cos \theta\left(x_{1}+x_{2}\right)\right)\right)}{\left(z_{1} x_{2}-z_{2} x_{1}\right)+(a-c)\left(\sin \theta\left(z_{1}+z_{2}\right)+\cos \theta\left(x_{1}-x_{2}\right)\right)-2(a-c)^{2} \sin \theta \cos \theta} \\
& z_{o}=\frac{x_{o} z_{1}-(a-c) x_{o} \cos \theta-(a-c) z_{1} \sin \theta+(a-c) x_{1} \cos \theta}{x_{1}-(a-c) \sin \theta}
\end{aligned}
$$

For a negative rotation through an angle $\theta_{2}$, the new source and detector center coordinates are $\mathrm{S}^{\prime \prime}\left(\mathrm{x}_{\mathrm{s} 2}, \mathrm{y}_{\mathrm{s} 2}, \mathrm{z}_{\mathrm{s} 2}\right)$ and $\mathrm{D}^{\prime \prime}\left(\mathrm{x}_{\mathrm{d} 2}, \mathrm{y}_{\mathrm{d} 2}, \mathrm{z}_{\mathrm{d} 2}\right)$ respectively. $\mathrm{O}^{\prime \prime} ;\left(\mathrm{x}_{2}, \mathrm{y}_{2}, \mathrm{z}_{2}\right)$ is the projection of the lesion center on the detector plane in this configuration.

We now have the following:

$$
\begin{aligned}
x_{s 2} & =(a-c) \sin \theta_{2} ; \quad y_{s 2}=0 ; \quad z_{s 2}=(a-c) \cos \theta_{2} \\
x_{d 2} & =-c \sin \theta_{2} ; \quad y_{d 2}=0 ; \quad z_{d 2}=-c \cos \theta_{2} \\
x_{2} & =L_{x 2} \cos \theta_{2}+x_{d 2} ; \quad y_{2}=L_{y 2} ; \quad z_{2}=z_{d 2}-L_{x 2} \sin \theta_{2} .
\end{aligned}
$$

The equation of line $\mathrm{S}^{\prime \prime} \mathrm{O}^{\prime \prime}$ is given by

$$
\begin{aligned}
& \frac{x_{o}-x_{s 2}}{x_{2}-x_{s 2}}=\frac{y_{o}-y_{s 2}}{y_{2}-y_{s 2}}=\frac{z_{o}-z_{s 2}}{z_{2}-z_{s 2}} \\
& \frac{x_{o}-(a-c) \sin \theta_{2}}{x_{2}-(a-c) \sin \theta_{2}}= \frac{z_{o}-(a-c) \cos \theta_{2}}{z_{2}-(a-c) \cos \theta_{2}} \\
& \text { Let } S=(a-c) \sin \theta_{2} \\
& \text { and } U=(a-c) \cos \theta_{2} \\
& \frac{x_{o}-S}{x_{2}-S}=\frac{z_{o}-U}{z_{2}-U} \\
& z_{o}=\frac{\left(x_{o}-S\right)\left(z_{2}-U\right)}{x_{2}-S}+U .
\end{aligned}
$$

We can solve (18) and (22) simultaneously for $\mathrm{x}_{\mathrm{o}}$ and $\mathrm{z}_{\mathrm{o}}$. We get (23) and (24), as shown at the top of the page. If the two stereo views are chosen to be symmetric about the $\mathrm{z}$ axis then $\theta_{1}=-\theta_{2}=\theta$ and

$$
\cos \theta_{1}=\cos \theta_{2}=\cos \theta \text { and } \sin \theta_{1}=-\sin \theta_{2}=\sin \theta \text {. }
$$

We now substitute for R, S, T, U to get (25) and (26) shown at the top of the page. To solve for $\mathrm{y}_{\mathrm{o}}$

$$
\begin{aligned}
& \frac{x_{o}-x_{s 1}}{x_{1}-x_{s 1}}=\frac{y_{o}-y_{s 1}}{y_{1}-y_{s 1}} \\
& \frac{x_{o}-(a-c) \sin \theta}{L_{x 1} \cos \theta+x_{d 1}-(a-c) \sin \theta}=\frac{y_{o}}{L_{y 1}} \\
& y_{o}=\frac{L_{y 1}\left(x_{o}-(a-c) \sin \theta\right)}{L_{x 1} \cos \theta+x_{d 1}-(a-c) \sin \theta} .
\end{aligned}
$$

Equations (25), (26), shown at the bottom of the page, and (29) are Section II, (1)-(3).

\section{REFERENCES}

[1] F. Scopinaro, O. Schillaci, M. Scarpini, P. L. Mingazzini, L. Di Macio, M. Banci, R. Danieli, M. Zerilli, M. R. Limiti, and A. Centi Colella, "Technitium-99m sestamibi: An indicator of breast cancer invasiveness," Eur. J. Nucl. Med., vol. 21, no. 9, pp. 984-987, 1994.

[2] J. A. Cutrone, L. S. Yospur, I. Khalkhali, J. Tolmos, A. Devito, L. Diggles, M. P. Vargas, P. Shitabata, and S. French, "Immunohistologic assessment of technetium-99m-MIBI uptake in benign and malignant breast lesions," J. Nucl. Med., vol. 39, no. 3, pp. 449-453, 1998.

[3] I. N. Weinberg, R. Pani, R. Pellegrini, F. Scopinaro, G. DeVincentis, A. Pergola, and A. Soluri, "Small lesion visualization in scintimammography," IEEE Trans. Nucl. Sci., vol. 44, pp. 1398-1402, June 1997.

[4] M. B. Williams, M. J. More, D. Narayanan, S. Majewski, A. G. Weisenberger, R. Wojcik, M. Stanton, W. Phillips, and A. Stewart, "Combined structural and functional imaging of the breast," Technol. Cancer Res. Treatment, vol. 1, no. 1, pp. 39-42, Feb. 2002.

[5] M. B. Williams, M. J. More, D. Narayanan, S. Majewski, B. Welch, and D. A. Kieper, "Phantom study of radiotracer concentration quantification in breast scintigraphy," IEEE Trans. Nucl. Sci., vol. 50, pp. 433-438, June 2003.

[6] S. H. Parker, J. D. Lovin, W. E. Jobe, J. M. Luethke, K. D. Hopper, W. F. Yakes, and B. J. Burke, "Stereotactic breast biopsy with a biopsy gun," Radiol., vol. 176, no. 3, pp. 741-747, Sept. 1990.

[7] L. Becker, D. Taves, L. McCurdy, G. Muscedere, S. Karlik, and S. Ward, "Stereotactic core biopsy of breast microcalcifications: Comparison of film versus digital mammography, both using an add-on unit," AJR Amer. J. Roentgenol., vol. 177, no. 6, pp. 1451-7, Dec. 2001.

[8] H. M. Verkooijen and Core Biopsy After Radiological Localization (COBRA) Study Group, "Diagnostic accuracy of stereotactic large-core needle biopsy for nonpalpable breast disease: results of a multicenter prospective study with $95 \%$ surgical confirmation," Int. J. Cancer, vol. 99, no. 6, pp. 853-9, June 20, 2002.

[9] M. B. Williams, D. Narayanan, M. J. More, P. J. Goodale, S. Majewski, and D. Kieper, "Analysis of position-dependent compton scatter in scintimammography with mild compression," in Proc. IEEE Nuclear Science Symp. Conf. Rec., Norfolk, VA, Nov. 2002.

[10] S. Majewski, D. Kieper, E. Curran, C. Keppel, B. Kross, A. Palumbo, V. Popov, A. G. Weisenberger, B. Welch, R. Wojcik, M. B. Williams, A. R. Goode, M. More, and G. Zhang, "Optimization of dedicated scintimammography procedure using detector prototypes and compressible phantoms," IEEE Trans. Nucl. Sci., vol. 48, pp. 822-829, June 2001.

[11] I. Khalkhali, F. S. Mishkin, L. E. Diggles, and S. R. Klein, "Radionuclide-guided stereotactic prebiopsy localization of nonpalpable breast lesions with normal mammograms," J. Nucl. Med., vol. 38, no. 7, pp. 1019-1022, 1997. 